Portland State University

PDXScholar

\title{
Stratification of Protein Expression across the Pancreatic Ductal Adenocarcinoma Disease Axis to Inform an Early Detection Platform
}

Sarah Bumatay

Portland State University

Follow this and additional works at: https://pdxscholar.library.pdx.edu/honorstheses

Let us know how access to this document benefits you.

\section{Recommended Citation}

Bumatay, Sarah, "Stratification of Protein Expression across the Pancreatic Ductal Adenocarcinoma Disease Axis to Inform an Early Detection Platform" (2019). University Honors Theses. Paper 664. https://doi.org/10.15760/honors.677

This Thesis is brought to you for free and open access. It has been accepted for inclusion in University Honors Theses by an authorized administrator of PDXScholar. Please contact us if we can make this document more accessible: pdxscholar@pdx.edu. 
Stratification of Protein Expression across the Pancreatic Ductal Adenocarcinoma Disease Axis

to Inform an Early Detection Platform

by

Sarah Bumatay

An undergraduate honors thesis in partial fulfillment of the requirements for the degree of

Bachelor of Sciences

In

University Honors,

Health Sciences,

and

Biology

Thesis Advisor

Melissa Wong

Portland State University

2019 


\begin{abstract}
Pancreatic ductal adenocarcinoma (PDAC) is an extremely deadly disease with a dismal long-term prognosis. Currently there is an $8 \%$ 5-year survival rate for PDAC patients, which is due in part to a lack of early detection methods (Siegal et al, 2017). Notably, the majority of patients present with late stage disease, when therapeutic treatment is not effective. Novel biomarkers that can facilitate early detection of PDAC are needed to catch cancer earlier when there are more treatment options. Our laboratory has identified an overlooked population of tumor cells in the peripheral blood of cancer patients across all stages of disease. These cells are fusion hybrids between macrophages and pancreatic tumor cells, and harbor properties of both parental cell types-these cells are called circulating hybrid cells (CHCs). CHCs express the epithelial protein cytokeratin (CK) and the pan-leukocyte epitope, CD45. We have determined their existence in greater numbers compared to conventionally-defined circulating tumor cells (CTCs) that express CK, but not CD45 in cancer patients. In addition, we identified CHCs in patients with precursor pathologies or precursor lesions across all different stages of PDAC, leading us to expect that specific protein expression across the disease axis could increase specificity of CHCs in an early detection assay. To support this, I will test the hypothesis that distinct protein expression defines different stages of disease in the pancreatic cancer continuum. In utilizing a disease-specific tissue microarray, and antibodies recognizing discrete proteins identified with immunofluorescence, I differentiated states of pancreatic epithelium from normal, pancreatitis, pre-cancer (PanIN1, PanIN2, PanIN3), and cancer.
\end{abstract}




\section{Introduction}

PDAC is a type of exocrine pancreatic cancer deriving from cells that line ducts in the pancreas (Stark, A., \& Eibl, G, 2015). Advanced stages of pancreatic cancer are present in around $50 \%$ of patients due to the disease's heterogeneity of genetic mutations and lack of distinctive symptoms (Adamska et al, 2017). The only reliably curative treatment for patients with pancreatic cancer is surgical resection; however, only precancerous or early stages of PDAC are considered resectable. In order to detect earlier stages of disease efforts must focus on identification of patients with precursors of PDAC such as pancreatic intraepithelial neoplasms (PanINs), mucinous cystic neoplasms (MCNs), and intraductal mucinous cystic neoplasms (IPMNs). Novel biomarkers are needed to detect these precursors, because the most commonly used FDA approved blood biomarker test for PDAC, the carbohydrate antigen 19-9 (CA19-9) (Becker et al, 2014), is not routinely effective. CA19-9 is a poor screening tool for PDAC as it is expressed in low prevalence within the general population and is unreliable as a sensitive readout. Another potential biomarker for PDAC are conventionally defined circulating tumor cells (CTCs) (Poruk et al, 2016). CTCs are identified in the blood of patients with malignant neoplasm, but in very low numbers and rarely in healthy control samples (Poruk et al, 2016. Therefore, CTC-based assays have failed.

A novel biomarker, identified in our laboratory, is a circulating tumor cell that is the product of tumor cell-macrophage cell fusion, referred to as circulating hybrid cell (CHC). These CHCs are of interest due to the discovery of their existence in patients with precancerous lesions as well as metastatic pancreatic cancer at levels greater than conventionally defined CTCs (Gast et al, 2018). Although CTCs are also found across PDAC stages, CHCs exist in numbers greater in magnitude than CTCs in the metastatic setting (Gast et al, 2018). Therefore, we hypothesize that distinct protein expression can define the various stages of pancreatic ductal adenocarcinoma. 
Recognizing there are distinct morphologic differences between the different stages of pancreatic disease, identification of discrete protein profiles that define each stage of cancer can be applied to assays for early detection. Similarly, Schelitter et al identified prognostic relationships between histopathology and molecular profile based on the morphologic stratification of PDACs through mutational status of the four driver genes, KRAS, CDKN2A/p16, SMAD4 and TP53 (Jones et al, 2008). To identify discrete disease-specific protein expression, we generated pancreatic tissue microarrays (TMA) that represented pathology across the disease continuum (normal, inflammation, PanIN, and PDAC). We then validated an extensive number of antibodies with potential differential protein expression, and applied these antibodies to the TMA. Utilizing an immunofluorescence approach, protein expression patterns were visualized from the TMA. Overall, protein expression displayed differential patterns in the epithelium across the disease axis. We identified a subset of antibodies that recognized epithelial cells, and/or cancer cells and therefore have potential to distinguish different stages of pathology across the PDAC disease axis to increase specificity of CHC's in early detection platforms. 


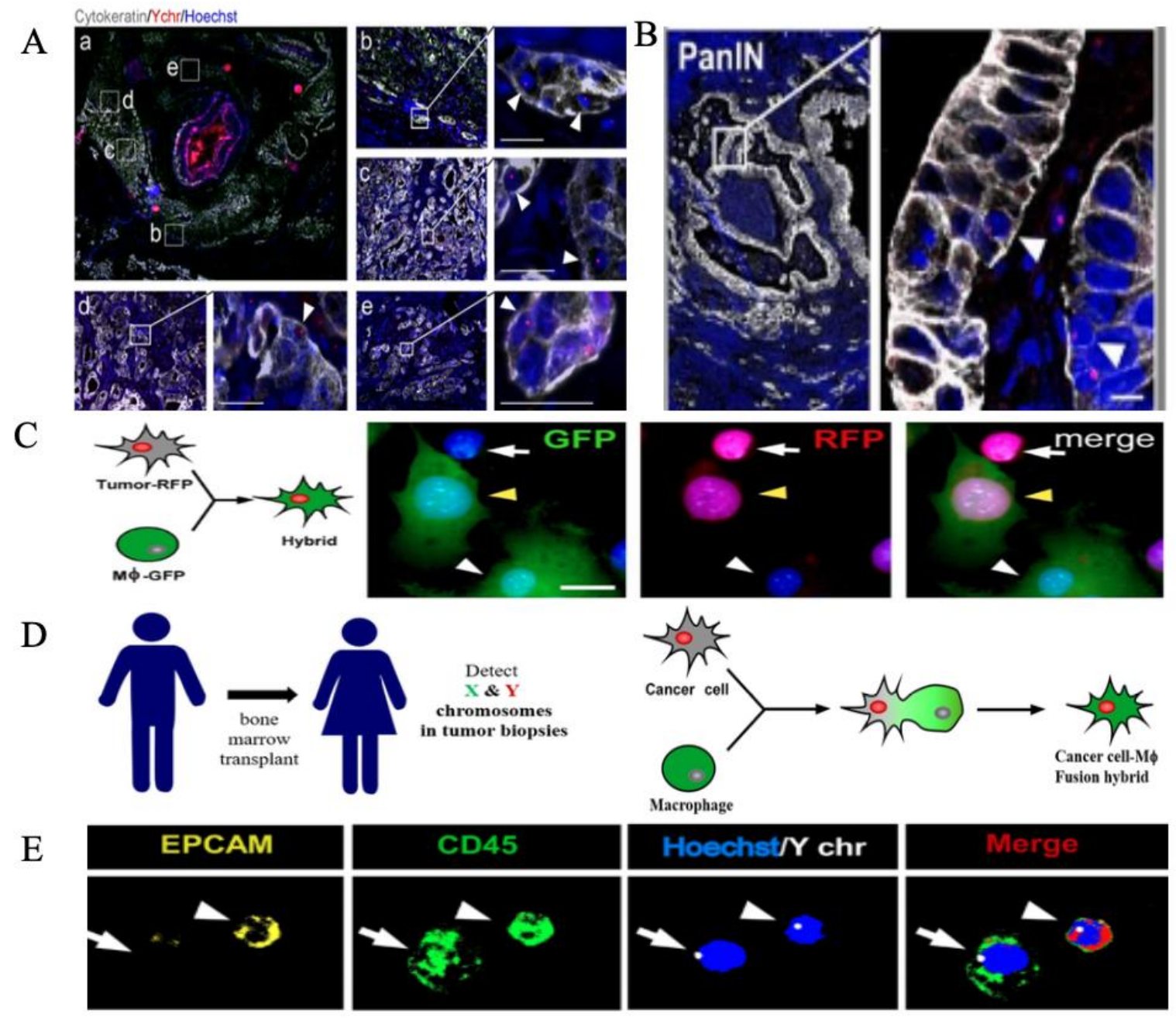

Figure 1: A, B) Human macrophage/tumor fusion: Identification of fusion hybrids in PDAC (left) and precancerous neoplasia (PanIN, right). Co-expression of Y-chromosome (red) and tumor epithelial marker cytokeratin (gray) demonstrates macrophage/tumor fusion

C, D) Diagram of macrophage fusion event. Schematic of macrophage fusion with cancer cells to create CHCs (C). GFP labeled macrophage fusing with RFP labeled tumor cell resulting in merged phenotype. Fusion event in YFP-Macrophages and RFP-Tumor cells (D). E) Cell fusion hybrids (arrowheads) that co-stain for EPCAM (yellow) and CD45 (green) and have a Y chromosome (white dot) in their nuclei (blue). Arrows denote leukocytes.

Reprinted/adapted from Gast, Charles E., et al. "Cell Fusion Potentiates Tumor Heterogeneity and Reveals Circulating Hybrid Cells That Correlate with Stage and Survival.” Science Advances, vol. 4, no. 9, 12 Sept. 2018, doi:10.1126/sciadv.aat7828. () The Authors, some rights reserved; exclusive licensee American Association for the Advancement of Science. Distributed under a Creative Commons Attribution NonCommercial License 4.0 (CC BY-NC) http://creativecommons.org/licenses/by-nc/4.0/ 
A

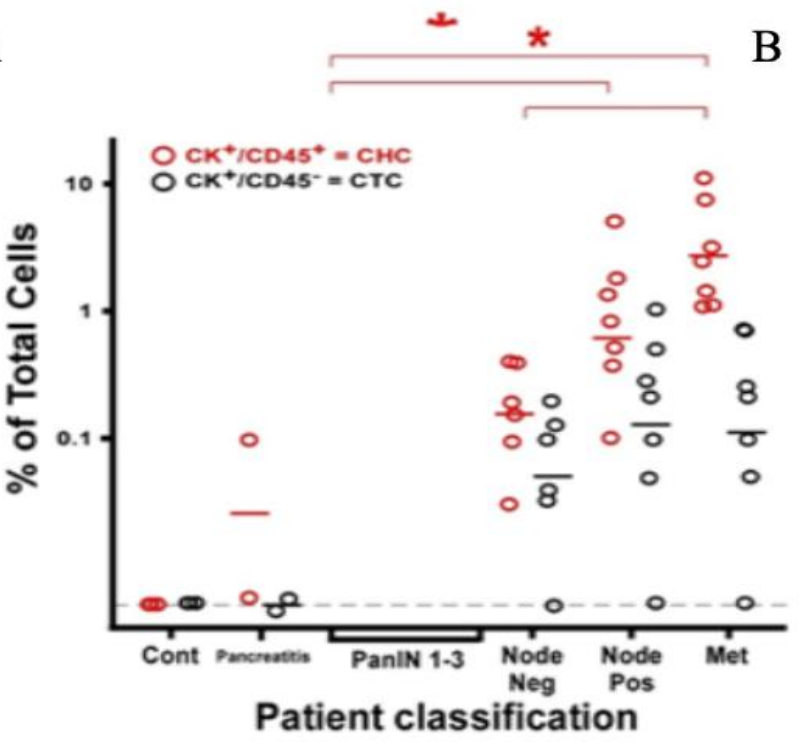

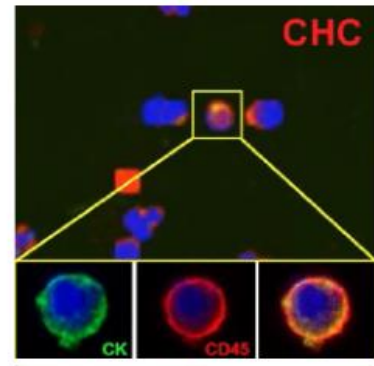

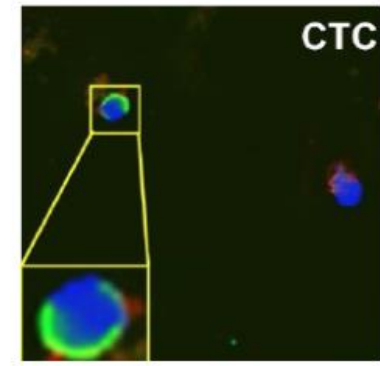

Figure 2: A) Quantification of CHCs and CTCs across the stages of PDAC development through flow cytometry. $\mathrm{CHCs}$ found in the precancerous setting as well as the node $-/+$ and metastatic setting.

B) Immunofluorescent identification of CHCs via the co-expression of tumor epithelial marker cytokeratin and panleukocyte marker CD45 $(\mathrm{CK}+/ \mathrm{CD} 45+$, left $)$ and $\mathrm{CTCs}$ (CK+/CD 45-, right). (Gast, et al, 2018).
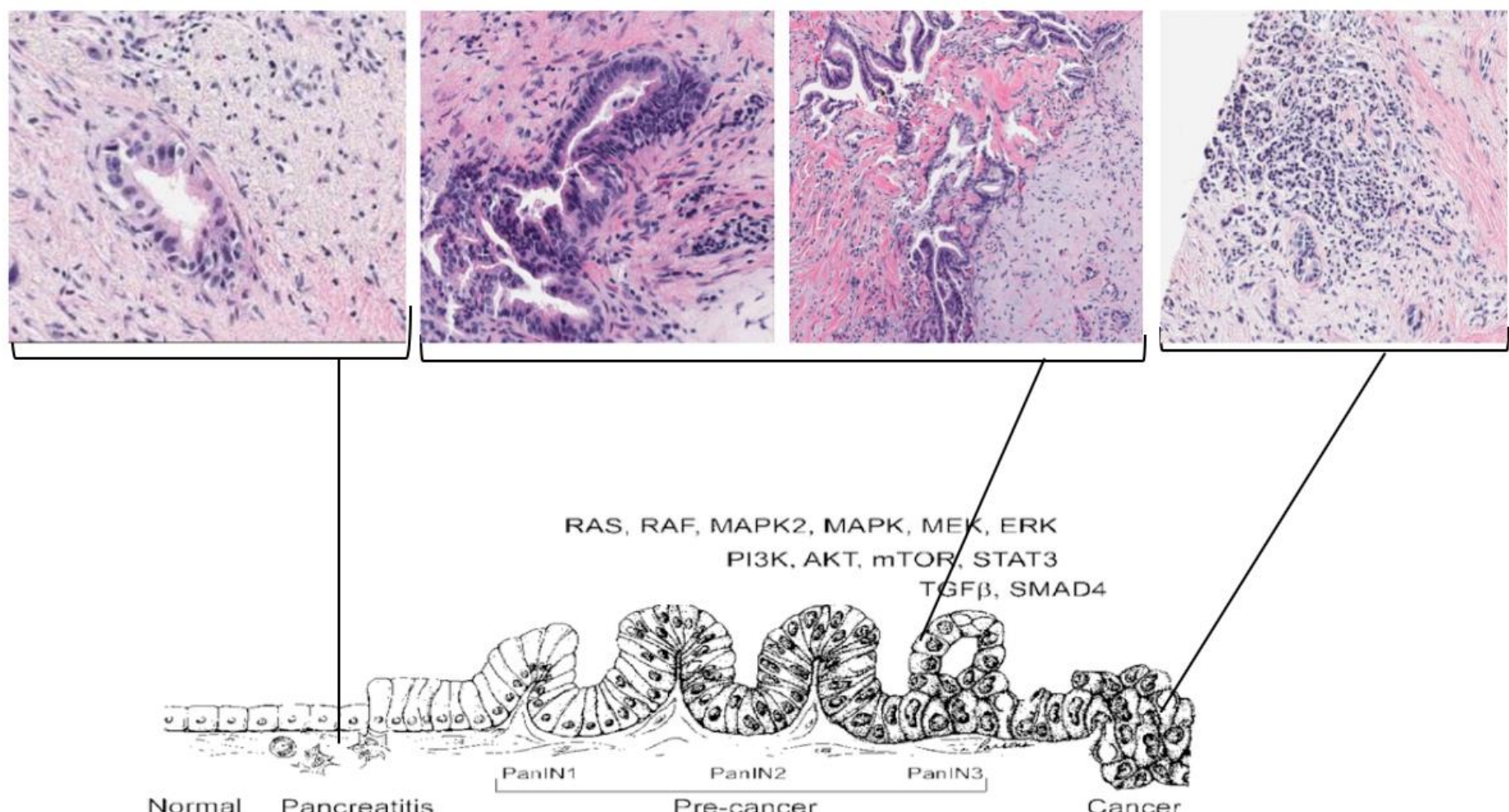

Figure 3: Pancreatic disease morphology (H\&E): Healthy to inflamed pancreatic tissue (A) characterized by circular duct structures. Progression through pancreatic intraepithelial neoplasia (PanIN) and PDAC results in increasing ductal irregularity and dysplasia (B,C,D).

Adapted from Clinical Cancer Research, 2000, Volume 6/Issue 8, 2969-2972, Ralph H. Hruban, Michael Goggins, Jennifer Parsons, Scott E. Kern, Progression Model for Pancreatic Cancer, with permission from AACR. Modifications by Melissa Wong. 


\section{Human Subjects/Ethics:}

\section{Materials and Methods}

Tissue samples were collected with approved protocols according to the ethical requirements and regulations of the Oregon Health and Science University (OHSU)

review board. All patients gave informed consent.

Generation of Tissue Microarray:

For tissue preparation, the selected FFPE tissues were marked for the specified tissue section utilized for the cores. A template for the TMA production was prepared with the tissues organized according to the outline:

A

\begin{tabular}{|l|l|l|l|l|l|l|l|l|}
\hline & & $\begin{array}{l}\text { Poor Diff } \\
\text { PDAC }\end{array}$ & $\begin{array}{l}\text { Mod Diff } \\
\text { PDAC }\end{array}$ & $\begin{array}{l}\text { Well Diff } \\
\text { PDAC }\end{array}$ & $\begin{array}{l}\text { Hi Grade } \\
\text { Pand N }\end{array}$ & $\begin{array}{l}\text { Low } \\
\text { PanlN }\end{array}$ & Normal & Pancreatitis \\
\hline Patient \# & a & 1 & 7 & 13 & 19 & 25 & 31 & 36 \\
\hline & b & 2 & 8 & 14 & 20 & 26 & 32 & 37 \\
\hline & c & 3 & 9 & 15 & 21 & 27 & 33 & \\
\hline & d & 4 & 10 & 16 & 22 & 28 & 34 & \\
\hline & e & 5 & 11 & 17 & 23 & 29 & 35 & \\
\hline & f & 6 & 12 & 18 & 24 & 30 & & \\
\hline & & 0 & 1 & 2 & 3 & 4 & 5 & 6 \\
\hline
\end{tabular}

B
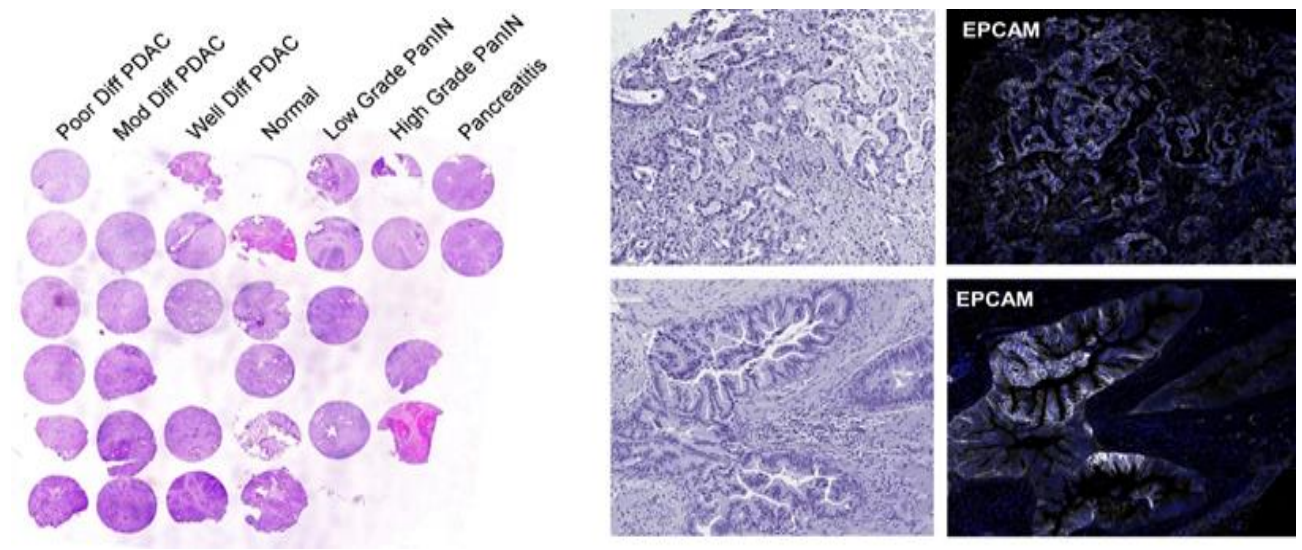

Figure 4: A) Tissue microarray (TMA) layout. Labels a-f and 0-6 were how we were able to designate what specific core/disease stage was being viewed under the microscope (6 cores per disease stage and 7 rows in total of the different stages). B) Hematoxylin and Eosin staining of TMA. 
For standardized sizing of the cores, a donor stylet would be marked at $4.5 \mathrm{~mm}$ with 2.5 $3 \mathrm{~mm}$ margins on each side of the paraffin block. Utilizing a manual tissue microarrayer, the selected punches were mounted and punched about $5 \mathrm{~mm}$ into a recipient block. The blocks were baked in $42{ }^{\circ} \mathrm{C}$ for 40 min then cooled for 10 min. The tissue block was cut into $4 \mu \mathrm{M}$ thick sections with a microtome consisting of a blade carrier with an integrated transfer bridge, heated water bath, and control unit. Sections were taken from the water bath immediately after being stretched out to be placed onto a superfrost glass slide. Once the slides are dried in room temperature over night, they are baked in $50{ }^{\circ} \mathrm{C}$ between 12 24 hours. Completed TMA slides were stained according to staining protocol described below.

\section{Paraffin Embedded PDAC Tissue IF and Imaging:}

Tissue samples were preserved by fixing in formalin to preserve the tissue. They are then embedded within a paraffin wax block to cut slices (about $10 \mu \mathrm{M}$ ) to mount on a microscopic slide. The tissue specimens that are obtained come from sections with PDAC present (tumor sections) and sections without (normal sections). The block of human tissue are excised from the patient and immersed in a neutral-buffered formalin solution for approximately 18-24 hours. In preparation for wax infiltration, the tissue would be dehydrated and cleared utilizing increasing concentrates of ethanol to then embed into IHC-grade paraffin. Formalin-fixed paraffin embedded (FFPE) specimens with $5 \mu \mathrm{m}$ sections of PDAC on glass slides were also ordered from Knight Biolibrary. The tissues were deparaffinized in three washes of xylene, $30 \mathrm{~min}, 10 \mathrm{~min}$, and $10 \mathrm{~min}$. Tissue was then rehydrated in isopropanol baths, $3 \mathrm{x}$ for 3 minutes each, then washed in $\mathrm{dH}_{2} \mathrm{O}$ for 5 min, and PBS 3x 3 min. Heat-mediated antigen retrieval was performed in citrate buffer (10 mM citric acid at $\mathrm{pH}$ 6) at $100{ }^{\circ} \mathrm{C}$ for $30 \mathrm{~min}$. Slides were cooled for $25 \mathrm{mins}$, and followed by PBS washes ( $3 \times 3 \mathrm{~min}$ ), and incubation in blocking buffer (5 g BSA, $200 \mu \mathrm{L}$ 
$2.5 \mathrm{M} \mathrm{CaCl}_{2}$, and $500 \mu \mathrm{L}$ triton $\left.\mathrm{x}-100\right)$ at $20^{\circ} \mathrm{C}$ for $30 \mathrm{~min}$. Primary antibodies were diluted in blocking buffer; Notch1 (Cell Signaling Technology, 1:100, 1:200, 1:500), EpCAM (Biolegend, 1,:100, 1:200, 1:500), Maspin (US Biological, 1:100, 1:200, 1:500), CK (ThermoFischer Scientific, 1:20, 1:30, 1:40), MUC4 (Millipore Sigma, 1:100, 1:250, 1:500), Glypican-1 (ThermoFischer Scientific, 1:50, 1:100, 1:200), BMI1 (Cell Signaling Technology, 1:100, 1:200, 1:500), MUC5 (ThermoFischer Scientific, 1:100, 1:200), SMAD4 (Abcam, 1:100, 1:200), SerpinB5 (Sigma Aldrich, 1:100, 1:200, 1:500), CXCR4 (Abcam, 1:100., 1:200, 1:300), Ras (New East Bio, 1:100, 1:200, 1:500), p53 (Abcam, 1:100. 1:200, 1:500), p27 (Abcam, 1:100, 1:200, 1:500), S100P (Fischer Scientific, 1:100, 1:200, 1:300), Erbb2 (Sigma Aldrich, 1:100, 1:200, 1:500), VHL (Abcam, 1:100, 1:200, 1:500), LHX1 (Origene, 1:100, 1:200, 1:300), IEX1 (ThermoFischer Scientific, 1:50, 1:100, 1:200), MUC1 (Origene, 1:100, 1:200, 1:500), IMP-3 (Agilent, 1:100, 1:200, 1:500), Ki67 (Abcam, 1:100, 1:200, 1:500), PhosphoERK (Labome, 1:100, 1:200, 1:500), Annexin-A10 (Novus Biologicals, 1:100, 1:200, 1:500). Tissue sections were incubated with $100 \mu \mathrm{L}$ of diluted antibody and were incubated for $12 \mathrm{~h}$ at $4{ }^{\circ} \mathrm{C}$. For secondary antibody application, tissues were washed with PBS, $3 \times 3$ min, prior to application of fluorescent secondary antibodies (1:500 donkey anti-rabbit Alexa Fluor 488, 1:500 goat anti mouse AlexaFluor 488, 1:500 donkey anti rabbit Cy3, and 1:500 goat anti mouse Cy3; all Jackson ImmunoResearch). Tissue was incubated for $1 \mathrm{~h}$ at $20^{\circ} \mathrm{C}$ and washed with PBS for 3 min before nuclear counterstaining with Hoescht for $10 \mathrm{~min}$. They are washed one last time with PBS for 3 min and mounted in n-propyl gallate or prolong gold. Fluorescent images were taken utilizing a Zeiss ObserverZ1 microscope. 


\section{Results}

Table 1: Antibody validation results.

\begin{tabular}{|c|c|c|c|c|}
\hline Antibody & Catalog \# & Expected Cellular Staining & Expected Stage/State & Result \\
\hline ECAD & 31955 & Cytoplasmic/membranous & Normal to pancreatic cancer & More cytoplasmic/membranous in PDAC and expresses around single cells on exocrine glands. \\
\hline EpCAM & 118206 & Cytoplasmic/membranous & Normal to pancreatic cancer & Remains specifically around epithelium within PDAC. \\
\hline CK & MA1-82041 & Cytoplasmic/membranous & Normal to pancreatic cancer & Cytoplasmic/membranous in PDAC with expression in exocrine glands of normal tissue. \\
\hline Maspin & M2412.50F & Cytoplasmic/membranous & Pancreatic cancer & Cytoplasmic/membranous with secretion into extracellular space in PDAC and no expression in normal tissue. \\
\hline MUCA & MABT395 & Cytoplasmic/membranous & Pancreatic cancer & Cytoplasmic/membranous in PDAC with no expression in normal. \\
\hline BMI1 & 58565 & Nuclear & Normal to pancreatic cancer & Nuclear expression in PDAC tissue with less prominance in normal tissue. \\
\hline Glypican1 & PA5-24972 & Cytoplasmic/membranous & Pancreatic cancer & Cytoplasmic/membranous in PDAC with no expression in normal. \\
\hline Notch1 & Val1744 & Cytoplasmic/nuclear & Normal to pancreatic cancer & More nuclear in PDAC and appeared more cytoplasmic/membranous in normal tissue. \\
\hline MUC5 & MA5-12178 & Cytoplasmic/membranous & Pancreatic cancer & Cytoplasmic and membranous in normal and PDAC tissue. \\
\hline SMAD4 & $\mathrm{ab} 40759$ & Cytoplasmic/membranous & Normal to pancreatic cancer & Cytoplasmic and membranous in PDAC tissue, yet appeared more nuclear in normal. Overall staining showed low specificity. \\
\hline CXCR4 & ab181020 & Membranous & Normal to pancreatic cancer & Did not appear as prominent in normal tissue and displayed membranous in PDAC. Overall staining showed low specificity. \\
\hline Ras & 26036 & Cytoplasmic/membranous & Normal to pancreatic cancer & Overall staining showed low specificity. \\
\hline p53 & $\mathrm{ab} 1101$ & Cytoplasmic/nuclear & Normal to pancreatic cancer & Appeared more membranous in normal and PDAC tissues. Had overall low specificity. \\
\hline p27 & ab206927 & Cytoplasmic/membranous & Predominately normal & Appeared more membranous in normal and PDAC tissues. Had overall low specificity. \\
\hline S100P & BDB610307 & Cytoplasmic/membranous, nuclear & Pancreatic cancer & No expression in normal tissue and did not have prominent expression in PDAC. \\
\hline ERBB2 & HPA001383 & Cytoplasmic/membranous & Pancreatic cancer & Cytoplasmic and membranous in normal and PDAC tissue. \\
\hline VHL & $a b 135576$ & Cytoplasmic/membranous & Normal and rare in pancreatic cancer & Did not display any expression. \\
\hline LHXI & TA504526 & Nuclear & Unknown & Showed no expression in normal or PDAC tissue. \\
\hline IEX] & PA5-48274 & Cytoplasmic/membranous & Normal to pancreatic cancer & Cytoplasmic and membranous in normal and PDAC tissue. \\
\hline MUC1 & um870008 & Cytoplasmic/membranous & Normal to pancreatic cancer & Cytoplasmic and membranous in normal and PDAC tissue. \\
\hline IMP3 & M362629.2 & Nuclear & Normal to pancreatic cancer & Did not display any expression. \\
\hline KLF4 & $a b 75486$ & Nuclear & Pancreatic cancer & Showed slight expression in normal and more cytoplasmic/memranous in PDAC tissue. \\
\hline Annexin A10 & NBP1.90156 & Nuclear & Pancreatic cancer & Showed nuclear expression in both normal and PDAC tissue. \\
\hline PhosphoERK & 91065 & Cytoplasmic/membranous, nuclear & Normal to pancreatic cancer & Cytoplasmic and membranous in PDAC tissue, yet appeared more nuclear in normal. Overall staining showed low specificity. \\
\hline Ki67 & $a b 15580$ & Cytoplasmic/membranous, nuclear & Pancreatic cancer & Showed slight expression in normal and more cytoplasmic/memranous in PDAC tissue. \\
\hline
\end{tabular}

After validating antibody dilution for optimal tissue staining, the following antibodies were initially determined to have potential for staining onto the tissue microarray slides: CK (1:40 dilution), EpCAM (1:200 dilution), ECAD (1:200 dilution), MUC4 (1:250 dilution), Maspin (1:100 dilution), BMI1 (1:500), Notch1 (1:100 dilition), and Glypican-1 (1:100 dilution).

ECAD has shown to display individualized cellular patterns alongside strong membranous expression of tumor and epithelial regions within normal and cancer tissue.

EpCAM displays membranous and cytoplasmic within tumor regions while also

expressing within the islets of Langerhans and exocrine glandular cells within normal

tissue. CK expresses more cytoplasmic in tumor tissue with higher expression in the

exocrine glandular cells in normal tissue. MUC4 displayed expression localized 
cytoplasmic/membranous in PDAC and did not display in normal tissue. Maspin has shown to express more cytoplasmic/membranous within PDAC tissue, yet also has instances of expresses nuclear as well. It does not express within the exocrine glandular cells in normal tissue. BMI1 expresses nuclear within tumor tissue, and has lower expression of the exocrine glandular cells and higher in islets of Langerhans in normal tissue. Glypican-1 stains cytoplasmic/membranous in PDAC tissue with no expression in normal tissue. Although Notch1 was of initial interest to apply onto a TMA, it did not give discrete protein profile expression.

Antibody staining of TMA:

Currently, MUC4 and ECAD have displayed differentiated expression throughout PDAC on the TMA slides. MUC4 has expressed throughout the pancreatic disease states, yet did not display in the Normal or Pancreatitis tissue cores. This indicates MUC4 as a precursor before differentiated PDAC. As for ECAD, it has shown expression throughout the disease states, with prominence of immunofluorescence in the advanced stages. Because of these antibody's discrete expression throughout the pancreatic cancer, these were the ones that have been captured so far utilizing digital microscopy. There has been some variance in the antigen retrieval step for the IHC protocol within certain antibodies, which needs to be solidified before applying the rest onto TMAs. In order to do so, different timings of antigen retrievals have been tested and will be continued alongside varying methods of the retrieval if time adjustments are deemed unnecessary. 
Immunohistochemistry Staining of Paraffin Embedded Formalin Fixed Pancreatic

Normal and PDAC Tissue
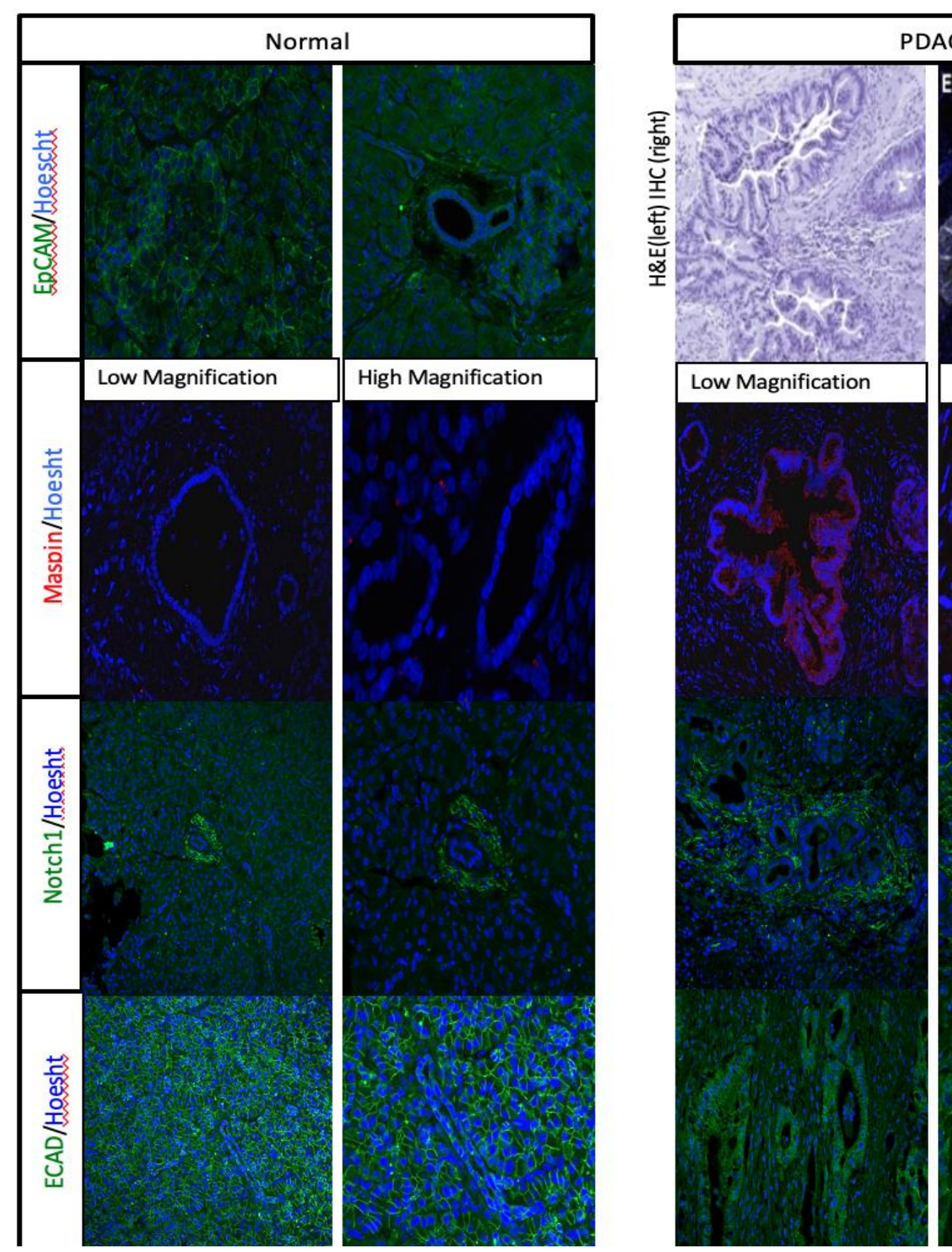

PDAC
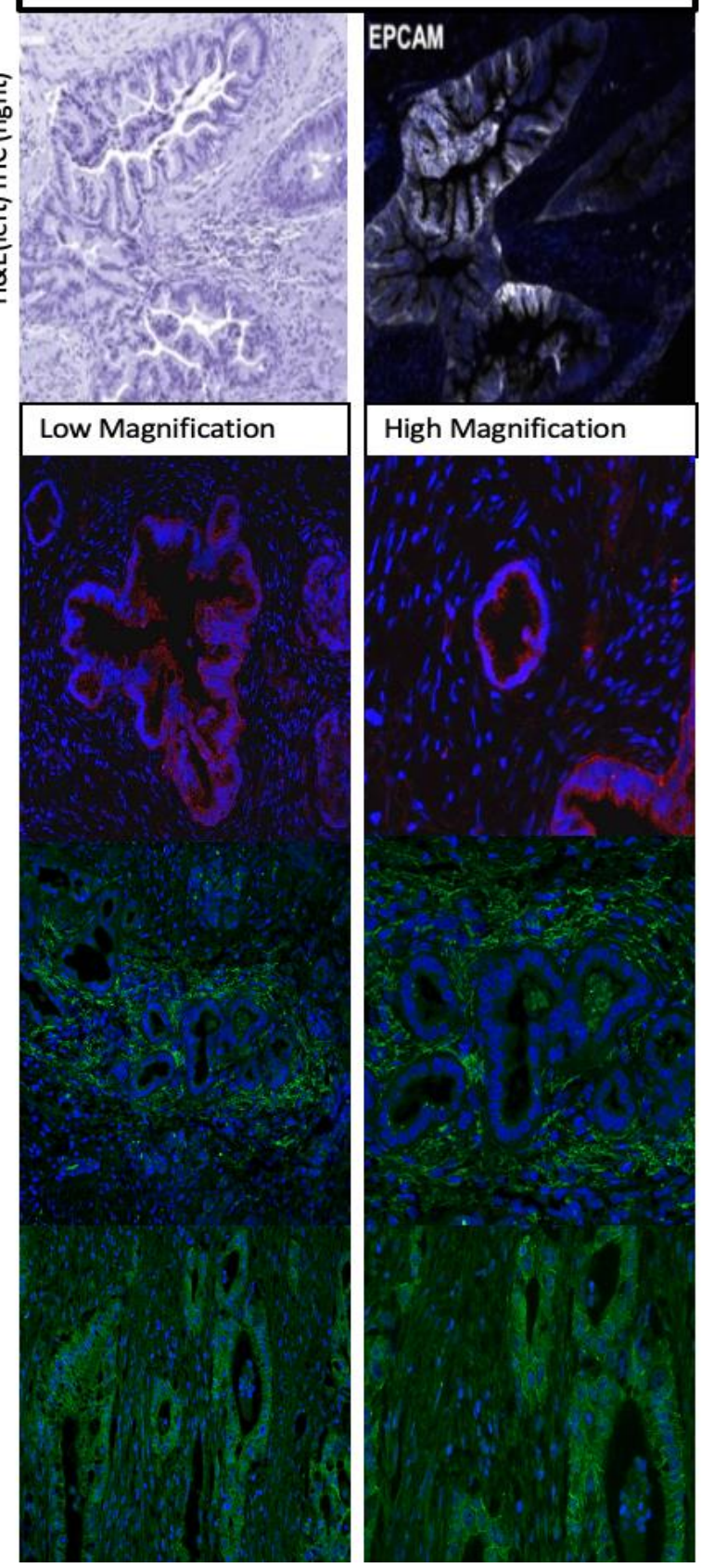

Figure 5: Preliminary staining across PDAC Continuum: Left is the normal tissue while right is PDAC tissue. These tissues have been costained with their respective protein markers and DAPI, which is a nuclear stain. EpCAM staining in PDAC remains specifically around the epithelium, which is also evident in H\&E staining (top left of PDAC). Maspin stains cytoplasmic/membranous and secretes into extracellular space in PDAC tissue, yet is not present in normal tissue. Notch1 appears to stain more nuclear in PDAC, and more apical cytoplasm and membranous in normal tissue. ECAD stains in both PDAC and normal tissue with individualized cellular expression on the cell surface. 
Immunohistochemistry Staining of Paraffin Embedded Formalin Fixed Pancreatic Tissue

Micro Array

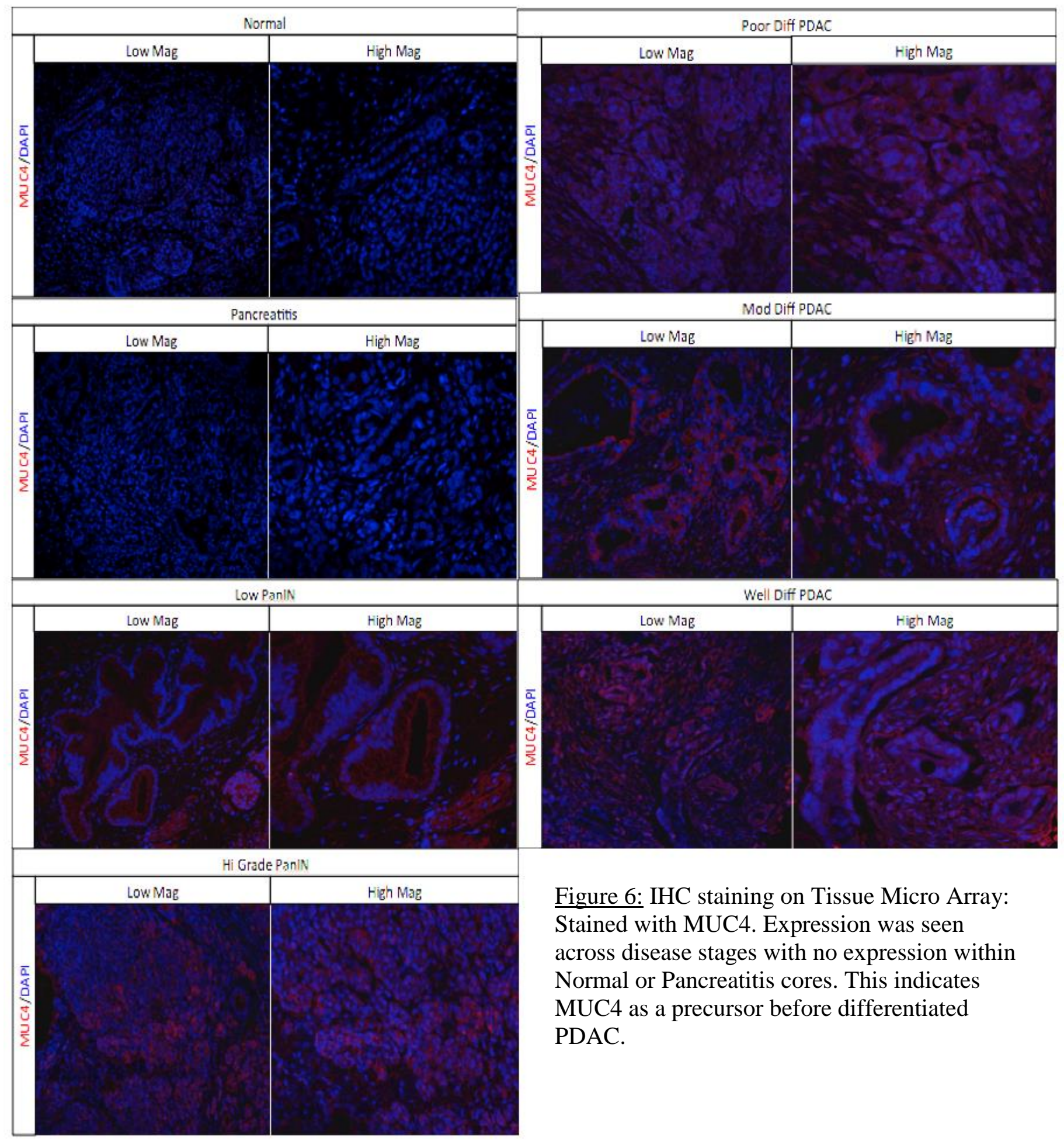




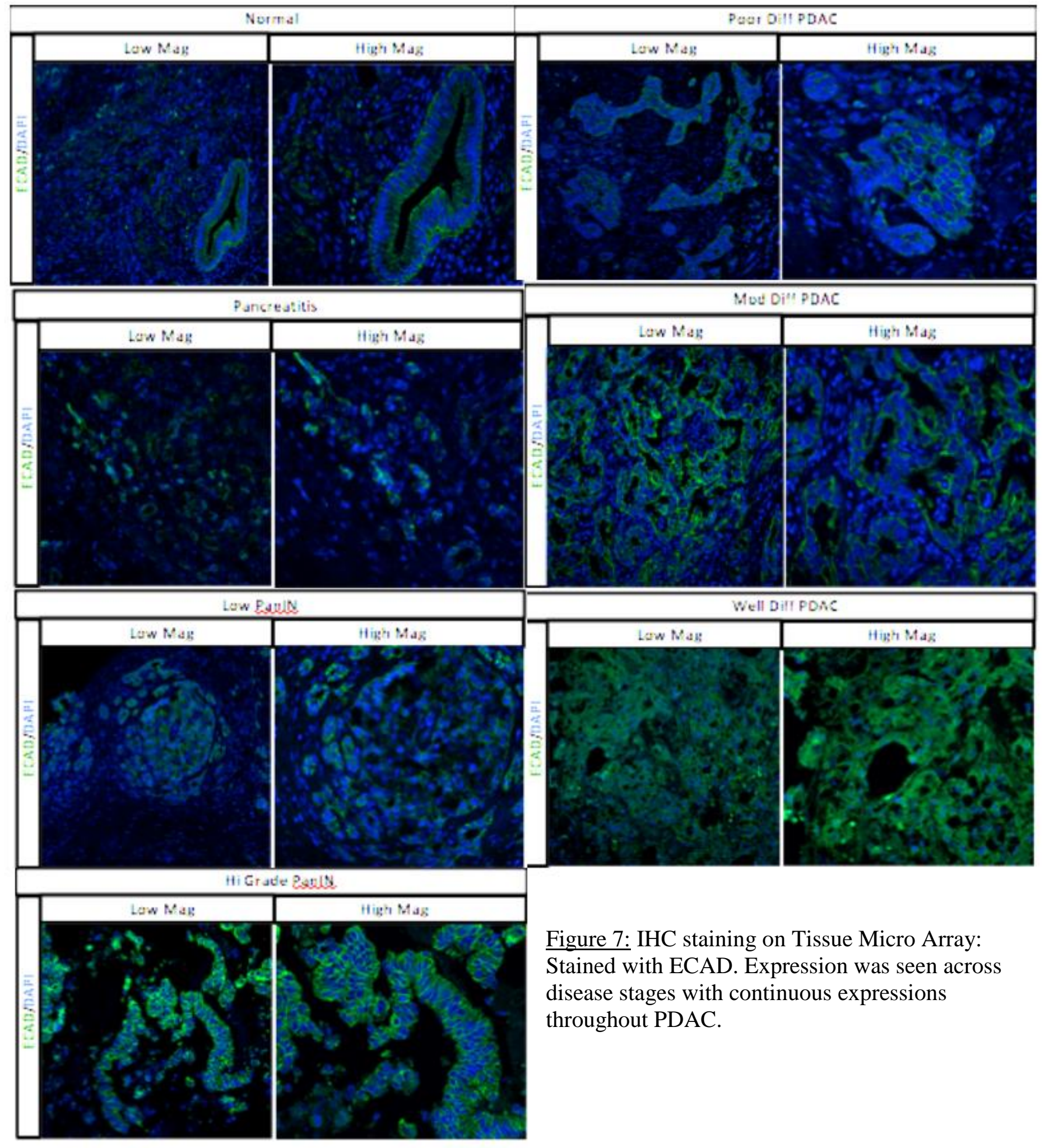




\section{Discussion}

Because PDAC is such an aggressive disease, gaining the ability to detect earlier stages is critical. Currently, early detection methods are dismal and early pancreatic cancers do not often cause any symptoms (Ryan et al, 2014). However, protein expression can change over progressive disease states. Identification of the morphological differences within disease stages alongside protein expression can help facilitate early detection.

Protein expression results have indicated increased expression of proteins such as MUC4, Bmi1, CD133, and CD166 throughout PDAC progression. These staining patterns varied across the phases of PanIN and thus display complex regulation of the epithelium across the disease axis. In this study, various antibodies were tested on PDAC tissue as well as normal tissue. Through IHC methods, we identified a subset of antibodies that displayed expression differences between PDAC tissue and normal tissue. For specific epithelial expression, ECAD, EpCAM, and CK were highly and uniformly expressed in all epithelia. For cancer specific protein expression, Maspin, BMI1, and Glypican-1 displayed strong expression. These antibodies have showcased specificities in their expression patterns between PDAC and normal tissue, thus the process of applying them to TMA slides have begun.

ECAD was selected for the panel due to findings that loss of ECAD can generate dedifferentiation of human carcinoma cells, thus being a tumor suppressor gene (Frixen, 1991). It has shown to express in pancreatic carcinoma cell lines. ECAD is an important molecule for cell-cell adhesion in epithelial tissues localizing on surfaces of epithelial cells within adheren junctions (Pećina-Slause, 2003), so it would be expected to see expression from normal tissue and throughout the stages of PDAC. EpCAM is an epithelial cell adhesion molecule that has also shown prominent expression within cancer 
stem cells (CSC) with frequent expression in various cancers, including pancreatic cancer (Gires et al, 2009). Cell adhesion is a regulator of cell polarity, thus affecting proliferation and differentiation for contact inhibition (Schnell et al, 2013). In also being an epithelial cell adhesion molecule, it is expected to be present in normal tissue to PDAC tissue. $\mathrm{CK}$ has been found to be abundant in epithelial cells alongside being useful markers for epithelial malignancies (Barak et al, 2004). There has been more than 20 cytokeratins identified through releasing from proliferating or apoptotic cells, which makes $\mathrm{CK}$ another marker found in normal tissue and earlier stages of pancreatic cancer (Barak et al, 2004). Maspin is a serine protease inhibitor that has displayed evidence of limiting metastases in various carcinomas, alongside expression in pancreatic cancer and not in normal pancreatic tissue (Nash et al, 2007). Because tumor metastasis requires invasion of tumor cells, increased adhesion through Maspin can be a contributor to inhibiting tumor metastases and displaying expression in late stages PanIn and PDAC (Berardi et al, 2013). BMI1 is a part of the polycomb protein family and controls histone ubiquitination and repression of genomic loci. It has shown upregulation in early PanINs (Schofield et al, 2015), and should be expected to be expressed throughout all stages of pancreatic cancer. Since BMI1 represses genes that induce cell death and senescence, it is required for maintaining adult stem cells in some tissues (Park et al, 2004). Lastly, Glypican-1 is a protein anchored to the cell membrane that has shown overexpression within pancreatic cancer (Melo et al, 2015). They are a member of heparan sulfate proteoglycans, which are abundant on cell surfaces, extracellular matrices, and connective tissues alongside being involved in cell recognition, growth, adhesion, proliferation, differentiation, and morphogenesis (Frampton et al, 2018). Because of these findings, alongside preliminary staining, all of these markers were selected to be applied onto TMA. 
Overall, healthy and PDAC regions are the only tissues that could be clearly identified on the basis of epithelial and CSC markers. In order to observe a more stage specific molecular signature, more PanIN specific markers must be targeted. Future plans at this point once classifying appropriate antibody expression in PDAC, are to continue utilizing the antibodies chosen for an Epithelial (ECAD, EpCAM, CK) and Cancer (Maspin, BMI1, Glypican-1) to be placed on tissue micro arrays as cocktails. By utilizing a disease-specific tissue microarray alongside protein markers based upon immunofluorescence, we are continuing to differentiate states of pancreatic epithelium from normal, pancreatitis, pre-cancer (PanIN1, PanIN2, PanIN3), and cancer.

In differentiating states of pancreatic epithelium, the antibody panels can be applied to patient CHCs. By quantifying CHCs and identifying a correlation between the prominence of CHCs, immunofluorescent expression, and disease stage, we can eventually create a more specific biomarker for pancreatic cancer detection. PDAC is one of the most lethal cancers with few treatments for patients, thus being the fourth common cause of cancer related deaths (Becker et al, 2014). 80-90\% present late stages of the disease that are surgically incurable (Stark et al, 2015). Only precancerous or early stage PDAC is considered surgically resectable (Becker et al, 2014), so the focus should be on improving methods for early detection - earlier stages are usually asymptomatic (Becker et al, 2014). By identifying and screening high-risk populations before their symptoms arise, detection of earlier-stage lesions that may still be at a surgically-resectable stage can increase survival rates. In working to discover an early detection method involving the analysis of patient blood, a more non-invasive method can be created and utilized to determine the status of disease at much earlier states. 


\section{Abbreviations}

- PDAC: Pancreatic ductal adenocarcinoma

- PanIN: Pancreatic intraepithelial neoplasm

- IPMN: Intraductal mucinous cystic neoplasm

- MCN: Mucinous cystic neoplasm

- CHC: Circulating hybrid cell

- CTC: Circulating tumor tell

- CSC: Cancer stem cell

- TMA: Tissue microarray

- IHC: Immunohistochemistry

- IF: Immunofluorescence

- FFPE: Formalin-fixed paraffin embedded 


\section{References}

Adamska, Aleksandra, Alice Domenichini, and Marco Falasca. "Pancreatic Ductal Adenocarcinoma: Current and Evolving Therapies.” International Journal of Molecular Sciences 18.7 (2017): 1338. PMC. Web. 31 July 2018.

Barak, V., Goike, H., Panaretakis, K. W., \& Einarsson, R. (2004). Clinical utility of cytokeratins as tumor markers. Clinical Biochemistry, 37(7), 529-540. doi:10.1016/j.clinbiochem.2004.05.009

Becker, A. E., Hernandez, Y. G., Frucht, H., \& Lucas, A. L. (2014). Pancreatic ductal adenocarcinoma: Risk factors, screening, and early detection. World Journal of Gastroenterology : WJG, 20(32), 11182-11198.

Berardi, R., Morgese, F., Onofri, A., Mazzanti, P., Pistelli, M., Ballatore, Z., Savini, A., De Lisa, M., Caramanti, M., Rinaldi, S., Pagliaretta, S., Santoni, M., Pierantoni, C., ... Cascinu, S. (2013). Role of maspin in cancer. Clinical and translational medicine, 2(1), 8. doi:10.1186/2001-1326-2-8

Frampton, A. E., Prado, M. M., López-Jiménez, E., Fajardo-Puerta, A. B., Jawad, Z., Lawton, P., Giovannetti, E., Habib, N. A., Castellano, L., Stebbing, J., Krell, J., ... Jiao, L. R. (2018). Glypican-1 is enriched in circulating-exosomes in pancreatic cancer and correlates with tumor burden. Oncotarget, 9(27), 1900619013. doi:10.18632/oncotarget. 24873

Frixen, U. H. (1991). E-cadherin-mediated cell-cell adhesion prevents invasiveness of human carcinoma cells. The Journal of Cell Biology, 113(1), 173-185. doi:10.1083/jcb.113.1.173

Gast, Charles E., et al. "Cell Fusion Potentiates Tumor Heterogeneity and Reveals Circulating Hybrid Cells That Correlate with Stage and Survival.” Science Advances, vol. 4, no. 9, 12 Sept. 2018, doi:10.1126/sciadv.aat7828. 
Kampf, C., Olsson, I., Ryberg, U., Sjöstedt, E., Pontén, F. Production of Tissue Microarrays, Immunohistochemistry Staining and Digitalization Within the Human Protein Atlas. J. Vis. Exp. (63), e3620, doi:10.3791/3620 (2012).

Kohler, I. , Bronsert, P., Timme, S. , Werner, M. , Brabletz, T. , Hopt, U. T., Schilling, O. , Bausch, D. , Keck, T. and Wellner, U. F. (2015), EMT in pancreatic cancer. J Gastroenterol Hepatol, 30: 78-84. doi:10.1111/jgh.12752

Melo, S. A., \& Kalluri, R. (2015). Glypican-1 identifies cancer exosomes and detects early pancreatic cancer. Nature, 177-182. doi:10.3410/f.725585052.793507726

Nash, J. W., Bhardwaj, A., Wen, P., \& Frankel, W. L. (2007). Maspin is Useful in the Distinction of Pancreatic Adenocarcinoma From Chronic Pancreatitis. Applied Immunohistochemistry \& Molecular Morphology, 15(1), 59-63. doi:10.1097/01.pai.0000203037.25791.21

Park, I. K., Morrison, S. J., \& Clarke, M. F. (2004). Bmi1, stem cells, and senescence regulation. The Journal of clinical investigation, 113(2), 175-9.

Paterlini-Brechot, P., \& Benali, N. L. (2007). Circulating tumor cells (CTC) detection: Clinical impact and future directions. Cancer Letters, 253(2), 180-204. doi:10.1016/j.canlet.2006.12.014

Pećina-Slaus N. (2003). Tumor suppressor gene E-cadherin and its role in normal and malignant cells. Cancer cell international, 3(1), 17. doi:10.1186/1475-2867-3-17

Poruk, K. E., Valero, V., Saunders, T., Blackford, A. L., Griffin, J. F., Poling, J., ... Wolfgang, C. L. (2016). Circulating Tumor Cell Phenotype Predicts Recurrence and Survival in Pancreatic Adenocarcinoma. Annals of Surgery, 264(6), 10731081.

Ryan, D. P., Hong, T. S., \& Bardeesy, N. (2014). Pancreatic Adenocarcinoma. The New England Journal of Medicine, 1039-1049. doi:10.1056/NEJMra1404198 
Schlitter, A. M., Segler, A., Steiger, K., Michalski, C. W., Jäger, C., Konukiewitz, B., ... Esposito, I. (2017). Molecular, morphological and survival analysis of 177 resected pancreatic ductal adenocarcinomas (PDACs): Identification of prognostic subtypes. Scientific Reports, 7, 41064.

Schnell, U., Cirulli, V., \& Giepmans, B. N. (2013). EpCAM: Structure and function in health and disease. Biochimica Et Biophysica Acta (BBA)Biomembranes, 1828(8), 1989-2001. doi:10.1016/j.bbamem.2013.04.018

Siegel, R.L., K.D. Miller, and A. Jemal, Cancer Statistics, 2017. CA Cancer J Clin, 2017. 67(1): p. 7-30.

Stark, Alexander. Eibl, Guido. (2015). Pancreatic Ductal Adenocarcinoma.

Pancreapedia: Exocrine Pancreas Knowledge Base, DOI: 10.3998/panc.2015.14

“Types of Pancreatic Cancer.” Pancreatic Cancer, Pancreatic Cancer UK, Aug. 2016. 